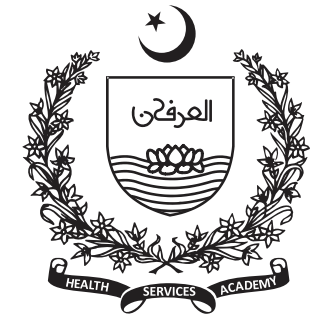

1Second Year MBBS Student, Aga Khan University, Karachi.

${ }^{2}$ Third Year MBBS Student, Aga Khan University, Karachi.

${ }^{3}$ Senior Instructor, Department of Community Health Sciences, Aga Khan University, Karachi

Corresponding Author: Malik Muhammad Hamza Khan

Email:

malik.hamza@scholar.aku.edu

\title{
Hooked on - The lure of internet addiction amidst Covid19
}

\author{
Malik Muhammad Hamza Khan ${ }^{1}$, Meher Angez², Maryam Pyar Ali Lakhdir ${ }^{3}$, \\ Ayesha Nasir Hameed ${ }^{2}$, Muhammad Talha Nawaz ${ }^{2}$
}

\section{Abstract}

Internet addiction has been seen to rise significantly during the SARS-CoV-2 pandemic especially during the quarantine period. Closure of offices, marketplaces, educational institutes, and lack of social interactions has led to an upsurge in the usage of the internet not only for academic and work but also for relaxation and a muddle through monotonic life amidst pandemic. It is essential to understand the effects of this addiction on the general population. Young's and Temperance's model; one of the behavioral models explain behavioral changes among individuals related to internet addiction amidst crisis. This paper aims to discuss how the intercalated interplay between biological characteristics such as brain plasticity, psychological and predisposing characteristics, environmental and need-based factors during the pandemic have led to internet addiction. This could help health authorities in developing effective methods to reduce internet addiction prevalence through preventive measures like counselling, therapeutic interventions, and a hotline service.

\section{Introduction}



ecember 2019 marked the month when the world got to know about SARS-CoV-2. Two months later, the World Health Organization (WHO) declared a global health emergency due to the emergence of a pandemic: the coronavirus (1). Ever since life for the majority has significantly changed: working from home, adhering to social isolation, closure of schools, universities, and marketplaces. Promotion of social distancing techniques and indoor activities to prevent the spread of the virus has also led to increased use of internet services to socialize, work, and have fun. Since the outbreak of the virus, research studies show that internet addiction has been on a constant rise ever since (2).

Understandably, internet addiction has emerged as a major concern during the pandemic expressing itself in people like depression, anxiety, and an altered mental status. This is due to people spending the majority of their time in isolation and having all their activities online. There is very less person to person contact and the constant rumors about the virus have contributed to an increasing sense of paranoia in the general public leading to problematic internet use and addiction (3). This is characterized by excessive or poorly controlled preoccupations, urges, or behaviors regarding computer use and Internet access (4). Besides, this leads to clinical signs and symptoms including compulsive use, withdrawal, tolerance, and adverse consequences, and comorbid conditions such as other substance use disorders, attention deficit hyperactivity disorder, anxiety, depression, and hostility (5). Thus, it is of prime importance to assess the prevalence, and reason for prevalence, of internet addiction during the SARSCoV-2 pandemic. This is to set the path for further research and then look into effective interventions that can be taken to curb this increasing addiction to the internet which has been shown to affect individuals in many harmful ways. Could internet addiction be the next pandemic of the century?

To assess internet addiction, there are some tools to diagnose, monitor, and explain behavioral changes. This is imperative because there is increasing evidence 
that people of all ages, particularly teens and young adults, are confronting genuine and sometimes serious outcomes in their day-to-day lives due to addictive utilization of the internet particularly during the pandemic. Concerning brain correlates, factors responsible for this addiction, and the majority of findings of internet addiction show commonalities to other behavioral addictions (6). According to Young's model, many factors predispose people to internet addiction one of which is overexposure to addictive substances such as the internet. Other contributing factors include stress, anxiety, and lack of social interactions. Furthermore, there could also be genetic and biological characteristics that further add to it (7). Looking further into the biological characteristics, addiction is a staggering compulsion that leads to alterations in brain chemistry affecting normal activities of the reward center. Addiction is mostly because of the unbalanced effects of the neurotransmitters: Dopamine and Serotonin. Withdrawal of the addictive substance leads to an imbalance within the brain causing symptoms typical of dependence. In addition, Internet overuse also severely affects the frontal cortex function and decreases cognitive functions leading to impulsive behavior.

There are some other models as well that further explain Young's model e.g.

Temperance Model and The Psychological or Character Logical Model. In line with these models, addiction is seen as an involuntary disease that the person is unaware of in the beginning (8). The person starts slowly and does not realize that he is becoming increasingly dependent on an easily accessible source of addiction. Since people are discouraged from going outdoors and meeting with other people in the Figure 1. This model explains the interaction between Bio-psychological constitutions, psychopathological factors, personality, social cognition, and environmental factors that reinforce internet addiction. A person's core characteristics include his personality, biopsychological constitution, psychopathology, and his motives. All of these characteristics have a huge role to play in how a person perceives and copes with the situation. Does he feel gratification? Does he stick by his decision? Does he feel like there is no other option? The answers to these questions could decide whether the person has a specific internet use disorder or not.

There is an urgent need to address the issues related to internet addiction and proper measures to reduce the pandemic, this has led to an increase in stress and anxiety levels (9). Moreover, everyone is seen as being vulnerable to addiction (8). However, some people are more prone to become addicted and the temperament traits are seen to be considerably greater in these patients. Also, certain age groups have been seen to show more addictive behavior such as teens and/or youth. This is for the reason that the majority of workload and responsibilities fall on this age group. Additionally, excessive use of a substance (internet) causes the brain to perceive it as pleasure or more desirable. Hence, increased use is seen as something necessary and not as something harmful (Figure 1).

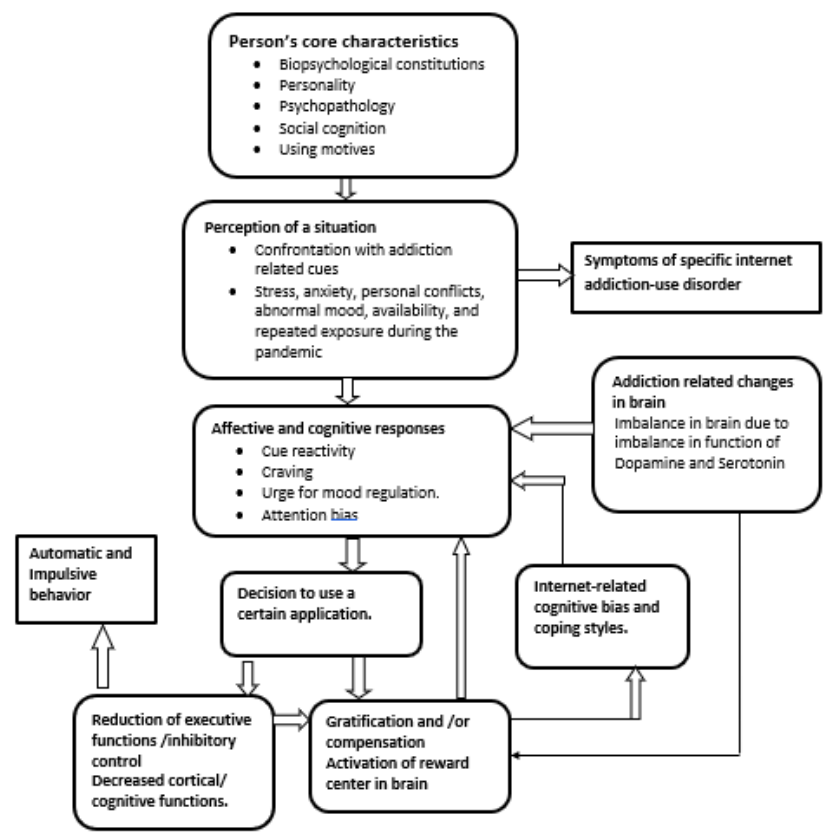

Figure 1. Temperance Model and The Psychological or Character Logical Model

prevalence of internet use should be established. Furthermore, there is a dire need to sensitize people about internet addiction-related issues. A hotline ought to be started to report and offer help and support for people suffering from internet addiction (10). Online counseling activities should be encouraged. Small groups of people should be allowed to go outside, and meet provided all of the necessary precautions are taken. Everyone who requires help or needs someone to talk to should be catered for; this needs to be implemented on a government level and not just on a personal level.

Supportive friends and a better work ethic could be some of the ways stress and anxiety levels are reduced, 
which ultimately could also contribute to decreased internet addiction levels. Lack of resources and awareness still pose to be problems but with better management and collaborations this issue can be brought to a decline. Educating individuals, having better alternatives to 'online work and entertainment' and finer coping mechanisms could be some of the ways internet addiction, stress and anxiety become less of an issue than they currently are in the SARS-CoV-2 pandemic.

Nonetheless, the question remains: 'Is the COVID pandemic leading to an internet addiction pandemic?'. Only time will tell.

\section{References}

1. Balkhair AA. COVID-19 Pandemic: A New Chapter in the History of Infectious Diseases. Oman Med J. 2020;35(2):e123.

2. Sun Y, Li Y, Bao Y, Meng S, Sun Y, Schumann G, et al. Brief Report: Increased Addictive Internet and Substance Use Behavior During the COVID-19 Pandemic in China. Am J Addict. 2020;

3. Király O, Potenza MN, Stein DJ, King DL, Hodgins DC, Saunders JB, et al. Preventing problematic internet use during the COVID-19 pandemic: Consensus guidance.
Compr Psychiatry. 2020/05/12. 2020 Jul;100:152180.

4. Weinstein A, Lejoyeux M. Internet Addiction or Excessive Internet Use. Am J Drug Alcohol Abuse. 2010 Aug;36(5):277-83.

5. Jorgenson AG, Hsiao RC-J, Yen C-F. Internet Addiction and Other Behavioral Addictions. Child Adolesc Psychiatr Clin N Am. 2016 Jul;25(3):509-20.

6. Young KS, Brand M. Merging theoretical models and therapy approaches in the context of Internet gaming disorder: A personal perspective. Front Psychol. 2017;8:1853.

7. Young KS. Internet addiction: The emergence of a new clinical disorder. Cyberpsychology Behav. 1998;1(3):23744.

8. Nora J. Models and Theories of Addiction and The Rehabilitation Counselor. Res Pap. 2013;1-25.

9. Garcia-Priego BA, Triana-Romero A, Pinto-Galvez SM, Duran-Ramos C, Salas-Nolasco O, Reyes MM, et al. Anxiety, depression, attitudes, and internet addiction during the initial phase of the 2019 coronavirus disease (COVID-19) epidemic: A cross-sectional study in Mexico. medRxiv. 2020;

10. Liese BS, Monley CM. Providing addiction services during a pandemic: Lessons learned from COVID-19. J Subst Abuse Treat. 2020;108156. 